\title{
The Effect of Color Correction of Endoscopy Images for Quantitative Analysis in Endometrium
}

\author{
M. S. Neophytou ${ }^{1,4}$, C. S. Pattichis ${ }^{1}$, V. Tanos ${ }^{2}$, M. S. Pattichis ${ }^{1,3}$, E. C. Kyriacou ${ }^{1}$, D. D. Koutsouris ${ }^{4}$ \\ ${ }^{1}$ Department of Computer Science, University of Cyprus, Nicosia, Cyprus, \\ \{mneoph, pattichi, mariosp, ekyriac\}@ucy.ac.cy \\ ${ }^{2}$ Evaggelistria Medical Center, Nicosia, Cyprus, tanosv@spidernet.com.cy \\ ${ }^{3}$ Deptartment of Electrical and Computer Engineering, University of New Mexico, NM, USA \\ ${ }^{4}$ Biomedical Engineering Laboratory, Department of Electrical and Computer Engineering, National Technical \\ University of Athens, Greece, dkoutsou@biomed.ntua.gr
}

\begin{abstract}
The objective of this study was to develop a standardized protocol for the capturing and analysis of endoscopy digital images for subsequent use in a Computer Aided Diagnosis (CAD) system in gynaecological cancer. Images were captured at optimum illumination and focus at $720 x 576$ pixels using 24 bits color in the following cases: (i) for a variety of testing targets from a color palette with known color distribution, (ii) different viewing angles and distances from calf endometrium, and (iii) images from the human endometrium. Images were then gamma corrected and their classification performance was compared against that of nongamma corrected images. No significant difference in texture features was found between the close up and panoramic views, and between angles, either before or after gamma correction. There was significant difference in certain texture features between normal and abnormal endometrium, both before and after gamma correction. Our findings suggest that proper color correction can significantly impact CAD system performance, and we recommend its application prior to quantitative texture analysis in gynaecological endoscopy.
\end{abstract}

Keywords-Endoscopy camera, gamma correction, gynaecological cancer, texture analysis, endometrium.

\section{INTRODUCTION}

$\mathrm{H}$ ysterocopy is considered the gold standard technique for diagnosis of intrauterine pathology [1]. The physician guides the telescope connected to a camera inside the human body in order to investigate suspicious lesions of cancer [2]. The objective of this study was to develop a standardized protocol for the capturing and analysis of hysteroscopy digital images for subsequent use in a Computer Aided Diagnosis (CAD) system in gynaecological cancer.

To the best of our knowledge, no similar study was carried out for hysteroscopy imaging. In previous work, we used experimental tissue, the abdominal cavity of a butcher chicken, comparing texture feature variability under different viewing conditions such as different angles (with 5 degrees difference) and different distances (at a distance of $3 \mathrm{~cm}$ in close up and $5 \mathrm{~cm}$ in panoramic) from the imaged object [3]. The results indicated that for small consecutive angles there is no significant difference in texture features analysis but there is significant difference when comparing panoramic vs. close up views [3]. In another study our group, demonstrated that some texture features can be used to differentiate between normal and abnormal endometrium images captured during hysteroscopy [4]. However, in both [3], [4], the images were not gamma corrected. Instead, image intensity normalization was addressed by the doctors by adjusting camera gain so that a medical band appears well balanced on the screen. This approach is bound to introduce many sources of variability in the acquisition. We investigate the use of gamma correction that allows us to standardize image acquisition, irrespective of the optical system, and study its effect on both image classification, and visual examination.

In this paper, in section II, the methodology followed in this study is described. Section III presents the results, and section IV the concluding remarks.

\section{Methodology}

\section{A. Recording of Video}

The CIRCON IP4.1 [5] endoscopic camera was used. The analog output signal of the camera (PAL 475 horizontal lines) was digitized at $720 \times 576$ pixels using 24 bits color at 25 frames per second, and was then saved in AVI format. The Digital Video Creator 120 frame grabber was used [6].

\section{B. Testing Targets}

The testing targets were obtained from the Edmund Industrial Optics Company [7]. Twenty four testing targets were selected from a color palette. Images were captured using the CIRCON camera and the corresponding targets were digitally generated based on the data given by the Edmund Optics Company. ROIs of $64 \times 64$ pixels were segmented for all colors except for black. The captured images and the digitally generated ones were used for computing the parameters of the gamma correction.

\section{Gamma Correction Algorithm}

Most of the cameras have a nonlinear relationship between the signal voltage and the light intensity [8]. The light intensity input to the endoscopy camera or the output of the display is proportional to the voltage raised to the power gamma as shown in equation (1).

$$
X_{\text {rec }}=c X_{\text {org }}^{\gamma}+b
$$

Where $X_{\text {rec }}$ is the recorded light-component intensity (red, green, or blue), $X_{\text {org }}$ corresponds to the original light- 
component intensity, $c$ is a gain factor, $b$ is either cutoff (camera) or black level light intensity, and $\gamma$ takes values in the range of 1 to 3 . Equation (1) is solved using non linear fitting in MATLAB [9] for computing $c, b$ and $\gamma$. This procedure is repeated for the red, green and blue components. When we have the values of $c, b$ and $\gamma$, the corrected output image is computed for the three components using:

$$
X_{c o r}=\left(\frac{X_{r e c}-b}{c}\right)^{1 / \gamma}
$$

Note that $X_{\text {org }}$ is replaced with $X_{c o r}$.

The Mean Square Error (MSE) was computed between the original and the recorded or gamma corrected images for each colour component using the following equation:

$$
M S E=\frac{1}{N^{*} M} \sum_{i, j=1}^{N, M}\left(X_{\operatorname{org}_{i, j}}-X_{r e c_{i, j}}\right)^{2}
$$

\section{Experimental Set Up and Material}

Using one calf endometrium we collected 10 sets of images under different viewing conditions: (i) close up vs. panoramic view (approximately at a distance of $4 \mathrm{~cm}$ in close up and $7 \mathrm{~cm}$ in panoramic view from the target) and (ii) angle1 vs. angle2 (with 2 degrees difference). The physician segmented manually the same ROI in all views (see Fig. 1).

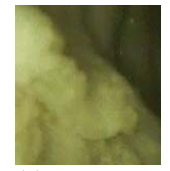

(a)

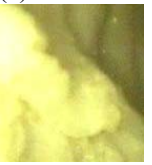

(f)

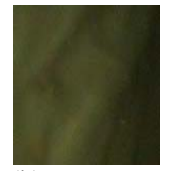

(b)

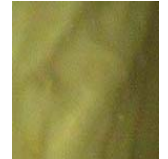

(g)

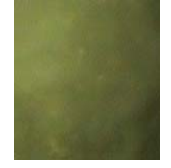

(c)

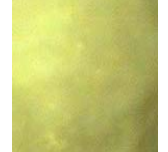

(h)

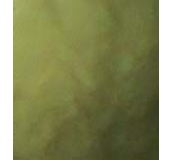

(d)

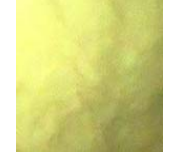

(i)
Fig.1. ROIs from the calf endometrium in different viewing conditions: (a) close up, (b) panoramic, (c) angle1, and (d) angle2, and the corresponding gamma corrected images in (f) - (i).

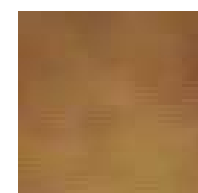

(a)

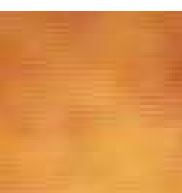

(b)

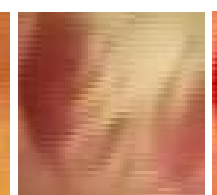

(c)

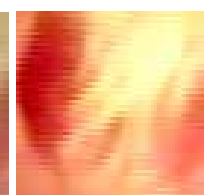

(d)
Fig.2. ROIs from the endometrium: (a) normal tissue, (b) normal tissue after gamma correction, (c) abnormal tissue, and (d) abnormal tissue after gamma correction.

In a different experiment using human endometrium a total of 25 ROIs of normal and 25 ROIs of abnormal endometrium were captured by the physician from three women. ROIs were of $64 \times 64$ pixels (see Fig. 2).

\section{E. Feature Extraction}

ROI images were transformed into grayscale and the following texture features [10] were computed:

Statistical Features (SF): SF features describe the gray level histogram distribution without considering spatial independence. The following texture features were computed: 1) Mean, 2) Variance, 3) Kurtosis and 4) Entropy.

Spatial Gray Level Dependence Matrices (SGLDM): The spatial gray level dependence matrices as proposed by Haralick et al. [11] are based on the estimation of the second-order joint conditional probability density functions that two pixels $(k, l)$ and $(m, n)$ with distance $d$ in direction specified by the angle $\theta$, have intensities of gray level $i$ and gray level $j$. Based on the estimated probability density functions, the following four texture measures out of the 13 proposed by Haralick et al. [11] were computed: 1) Contrast, 2) Correlation, 3) Homogeneity, and 4) Entropy. For a chosen distance $d$ (in this work $d=1$ was used), and for angles $\theta=0^{\circ}, 45^{\circ}, 90^{\circ}$ and $135^{\circ}$ we computed four values for each of the above texture measures. The above features were calculated for displacements $\delta=(0,1),(1,1)$, $(1,0),(1,-1)$, where $\delta \equiv(\Delta x, \Delta y)$, and their range of values were computed.

\section{RESULTS}

The values of $c, b$ and $\gamma$, were computed solving (1) with the captured testing targets as $X_{r e c}$, and the computer generated testing targets as $X_{\text {org }}$.

Table I tabulates these values for the CIRCON camera for the red, green, and blue components. Table II tabulates

TABLE I

GAMMA, C AND B FOR THE 3 COMPONENTS OF THE CIRCON ENDOSCOPY CAMERA

\begin{tabular}{cccc}
\hline Color component & c & b & $\gamma$ \\
\hline RED & 0.057 & 30.599 & 1.423 \\
GREEN & 0.111 & 40.609 & 1.302 \\
BLUE & 0.217 & 28.049 & 1.200
\end{tabular}

the MSE between $X_{\text {org }}$ and $X_{\text {rec }}$ for the non gamma corrected images and also the MSE between $X_{\text {org }}$ and $X_{c o r}$ for the gamma corrected images for the three components for the 24 testing targets. TABLE II

MSE BETWEEN THE ORIGINAL AND THE RECORDED IMAGE AND MSE BETWEEN THE ORIGINAL AND THE GAMMA CORRECTED IMAGES FOR THE 24 TESTING TARGETS.

\begin{tabular}{ccc}
\hline Color & MSE & MSE \\
Components & BETWEEN & BETWEEN \\
& $X_{\text {org }}-X_{\text {rec }}$ & $X_{\text {org }}-X_{\text {cor }}$ \\
\hline RED & 3342 & 1349 \\
GREEN & 2088 & 1034 \\
BLUE & 1228 & 880 \\
\hline
\end{tabular}

The MSE was of-course reduced after applying gamma correction. Using the values of $c, b$ and $\gamma$, the images captured for that particular session, images were corrected using (2). 
TABLE III

TEXTURE FEATURES FOR THE GAMMA CORRECTED CLOSE UP AND PANORAMIC VIEWS OF THE CALF

ENDOMETRIUM AND THE STATISTICAL ANALYSIS (WILCOXON RANK SUM TEST WITH ' 1 ' AND ' 0 ' INDICATING SIGNIFICANT AND NO SIGNIFICANT DIFFERENCE AT A=0.05, RESPECTIVELY) BETWEEN THE CLOSE UP AND PANORAMIC VIEWS.

\begin{tabular}{cccccccccc}
\hline & \multicolumn{4}{c}{ Close Up View } & \multicolumn{7}{c}{ Panoramic View } \\
\cline { 2 - 10 } & $\mathbf{Q}_{\mathbf{1}}$ & \multicolumn{1}{c}{ Median } & $\mathbf{Q}_{\mathbf{3}}$ & SIQR & $\mathbf{Q}_{\mathbf{1}}$ & Median & $\mathbf{Q}_{\mathbf{3}}$ & SIQR & H \\
\hline SF & & & & & & & & & \\
\hline MEAN & 41,91 & 80,65 & 125,33 & 41,71 & 42,42 & 50,73 & 58,72 & 8,15 & 0 \\
VARIANCE & 259,8 & 633,07 & 1708,22 & 724,21 & 108,13 & 201,17 & 244,69 & 68,28 & 0 \\
KURTOSIS & 1,91 & 2,33 & 2,77 & 0,43 & 2,31 & 2,56 & 2,66 & 0,18 & 0 \\
ENTROPY & 4,08 & 4,51 & 4,94 & 0,43 & 3,74 & 4,03 & 4,06 & 0,16 & 0 \\
\hline SGLDM & & & & & & & & & \\
\hline CONTRAST & 28,06 & 29,68 & 34,68 & 3,31 & 27,5 & 28,04 & 30 & 1,25 & 0 \\
CORRELATION & 0,95 & 0,97 & 0,98 & 0,02 & 0,87 & 0,92 & 0,94 & 0,04 & 0 \\
HOMOGENEITY & 0,19 & 0,21 & 0,21 & 0,01 & 0,2 & 0,21 & 0,21 & 0 & 0 \\
ENTROPY & 7,08 & 7,55 & 7,98 & 0,45 & 6,72 & 7,04 & 7,13 & 0,2 & 0 \\
\hline
\end{tabular}

NOTE1: THERE WAS NO SIGNIFICANT DIFFERENCE IN THE TEXTURE FEATURES BETWEEN THE CLOSE UP ORIGINAL AND THE GAMMA CORRECTED CLOSE UP VIEWS AS WELL AS BETWEEN THE PANORAMIC ORIGINAL AND THE GAMMA CORRECTED PANORAMIC VIEWS.

NOTE2: $\mathrm{Q}_{1}=$ LOWER QUARTILE (25\% PERCENTILE), $\mathrm{Q}_{3}=$ UPPER QUARTILE (75\% PERCENTILE), SIQR= SEMI INTER QUARTILE RANGE.

TABLE IV

TEXTURE FEATURES FOR THE GAMMA CORRECTED CONSECUTIVE ANGLES (ANGLE1, AND ANGLE2) OF THE CALF ENDOMETRIUM AND THE STATISTICAL ANALYSIS (WILCOXON RANK SUM TEST WITH '1' AND '0' INDICATING SIGNIFICANT AND NO SIGNIFICANT DIFFERENCE AT A=0.05, RESPECTIVELY) BETWEEN THE TWO ANGLES.

\begin{tabular}{|c|c|c|c|c|c|c|c|c|c|}
\hline & \multicolumn{4}{|c|}{ Angle 1 } & \multicolumn{4}{|c|}{ Angle 2} & \multirow[b]{2}{*}{$\mathbf{H}$} \\
\hline & $\mathbf{Q}_{1}$ & Median & $\mathbf{Q}_{3}$ & SIQR & $\mathbf{Q}_{1}$ & Median & $\mathbf{Q}_{3}$ & SIQR & \\
\hline \multicolumn{10}{|l|}{ SF } \\
\hline MEAN & 90,896 & 113,69 & 182,277 & 34,294 & 86,23 & 99,15 & 131,179 & 16,015 & 0 \\
\hline VARIANCE & 152,995 & 485,896 & 854,759 & 184,431 & 189,677 & 486,673 & 1123,464 & 318,396 & 0 \\
\hline KURTOSIS & 2,341 & 2,54 & 2,82 & 0,14 & 2,081 & 2,386 & 2,587 & 0,1 & 0 \\
\hline ENTROPY & 3,906 & 4,395 & 4,709 & 0,157 & 4,005 & 4,372 & 4,621 & 0,125 & 0 \\
\hline \multicolumn{10}{|l|}{ SGLDM } \\
\hline CONTRAST & 60,352 & 80,615 & 88,414 & 3,9 & 72,102 & 83,781 & 97,461 & 6,84 & 0 \\
\hline CORRELATION & 0,824 & 0,895 & 0,964 & 0,035 & 0,793 & 0,884 & 0,968 & 0,042 & 0 \\
\hline HOMOGENEITY & 0,13 & 0,136 & 0,154 & 0,009 & 0,126 & 0,135 & 0,143 & 0,004 & 0 \\
\hline ENTROPY & 7,378 & 7,854 & 8,073 & 0,109 & 7,522 & 7,852 & 7,951 & 0,05 & 0 \\
\hline
\end{tabular}

NOTE1: THERE WAS NO SIGNIFICANT DIFFERENCE IN THE TEXTURE FEATURES BETWEEN BOTH ANGLE1 AND ANGLE2 ORIGINALS AND THE CORRESPONDING GAMMA CORRECTED ANGLE1 AND ANGLE2 VIEWS.

NOTE2: $Q_{1}=$ LOWER QUARTILE (25\% PERCENTILE), $\mathrm{Q}_{3}=$ UPPER QUARTILE (75\% PERCENTILE), SIQR= SEMI INTER QUARTILE RANGE.

TABLE V

TEXTURE FEATURES FOR NORMAL AND ABNORMAL ENDOMETRIUM AND THE STATISTICAL ANALYSIS (WILCOXON RANK SUM TEST WITH ' 1 ' AND ' 0 ' INDICATING SIGNIFICANT AND NO SIGNIFICANT DIFFERENCE AT A=0.05, RESPECTIVELY) BETWEEN NORMAL ABNORMAL AND THE CORRESPONDING GAMMA CORRECTED IMAGES.

\begin{tabular}{|c|c|c|c|c|c|c|c|c|}
\hline \multirow{3}{*}{$\begin{array}{c}\text { Texture Features } \\
\text { Algorithms }\end{array}$} & \multicolumn{2}{|c|}{ Original images } & \multirow[b]{3}{*}{$\mathbf{H}$} & \multicolumn{2}{|c|}{ Corrected images } & \multirow[b]{3}{*}{$\mathbf{H}$} & \multirow{2}{*}{$\begin{array}{c}\text { Normal } \\
\text { Original vs } \\
\text { Gamma Corrected } \\
\end{array}$} & \multirow{2}{*}{$\begin{array}{c}\text { Abnormal } \\
\text { Original vs } \\
\text { Gamma Corrected } \\
\end{array}$} \\
\hline & Normal & Abnormal & & Normal & Abnormal & & & \\
\hline & \multicolumn{2}{|c|}{ Median Values } & & \multicolumn{2}{|c|}{ Median Values } & & $\mathbf{H}$ & $\mathbf{H}$ \\
\hline \multicolumn{9}{|l|}{ SF } \\
\hline MEAN & 82,08 & 106,73 & 1 & 100,26 & 141,18 & 1 & 1 & 1 \\
\hline VARIANCE & 56,76 & 369,87 & 1 & 184,24 & 697,44 & 1 & 1 & 1 \\
\hline KURTOSIS & 2,63 & 2,58 & 0 & 2,6 & 2,61 & 0 & 0 & 0 \\
\hline ENTROPY & 3,35 & 4,24 & 1 & 3,94 & 4,55 & 1 & 0 & 1 \\
\hline \multicolumn{9}{|l|}{ SGLDM } \\
\hline CONTRAST & 9,8 & 29,61 & 1 & 29,53 & 80,51 & 1 & 1 & 1 \\
\hline CORRELATION & 0,91 & 0,96 & 1 & 0,91 & 0,96 & 1 & 0 & 0 \\
\hline HOMOGENEITY & 0,37 & 0,26 & 1 & 0,27 & 0,22 & 0 & 1 & 1 \\
\hline ENTROPY & 5,69 & 6,86 & 1 & 6,67 & 7,42 & 1 & 1 & 1 \\
\hline
\end{tabular}


Figure 1 illustrates the original images captured from calf endometrium in the cases of close up and panoramic views and angle1 and angle2, whereas Fig. 2 illustrates two cases of normal and abnormal human endometrium real images. In both figures, the corresponding images after gamma correction are shown. The gamma corrected color images were visually better than the originals.

Tables III and IV tabulate the texture features for the calf endometrium for the gamma corrected close up and panoramic views, angle1 and angle2 respectively. It is clearly shown that there was no significant difference between the close up and panoramic views and between angle 1 and angle2. It is noted that the same applies for the original images. Also, there was no significant difference between the originals and gamma corrected images in all cases (close up, panoramic, angle1 and angle2).

Table $\mathrm{V}$ tabulates the median values of the $\mathrm{SF}$ and SGLDM texture features for the endometrium before and after gamma correction. There was a significant difference between normal and abnormal endometrium, both between the original and gamma corrected images. Also, the statistical results of the comparisons between original and gamma corrected images; for both normal and abnormal tissue illustrate that there was a significant difference in most of the texture features (see last two columns of Table $\mathrm{V}$ respectively).

1. Calibrate the endoscopy camera following the guidelines by the manufacturer (i.e. white balance).

2. Capture the 24 color ROIs using the color palette and digitally generated based on the data given by the manufacturer.

3. Compute the $c, b$ and $\gamma$, coefficients solving equation 1 .

4. Perform the endoscopy examination, identifying the anatomy of the organ and capture ROIs of tissue under investigation.

5. Gamma correct the ROI images using equation 2 and to visually assess the gamma corrected ROI.

6. Compute texture features on the gamma corrected images of step 5 .

7. Compare texture features of step 5 to corresponding digital library ones with normal and abnormal values and derive if there is significant difference or not.

8. Apply the computed texture features in a CAD system to assess the class of the tissue under investigation.

9. Carry out overall tissue assessment based on visual examination and steps 7 and 8

10. Repeat steps 4 to 9 as required.

Fig. 3 Proposed protocol for quantitative image analysis in gynaecological endoscopy.

\section{CONCLUding REMARKS}

Concluding, results showed that the gamma corrected color images were visually better than the originals. There was no significant difference in texture features between the close up and panoramic views, and between angles, either before or after gamma correction. There was significant difference in certain texture features between normal and abnormal endometrium, both before and after gamma correction. Based on these findings, a standardized protocol for the capturing and analysis of endoscopy digital images for subsequent use in a CAD system in gynaecological cancer is proposed (see Fig. 3).

It is anticipated that the standardized capturing and analysis of endoscopy images will facilitate the creation of digital libraries of endoscopy images for a variety of tissues in gynaecological cancer including the endometrium, the ovary, the cervix and other. These images could be captured in different endoscopy clinics, not only under different sessions from the same endoscopy imaging system (i.e. telescope, light source) but with different endoscopy cameras and different endoscopy imaging systems. Although for the capturing of images in the latter two set ups, further validation studies will be required.

Future work will capture images from more cases, as well as, investigate multiscale texture analysis, and the development of a CAD system based on neural network and statistical classifiers.

\section{ACKNOWLEDGMENTS}

This study is funded through the Research Promotion Foundation, Cyprus, PENEK 2002, Program for the Financial Support of New Researchers, through the project entitled: Integrated System for the Digital Analysis of Endoscopic Imaging Macrobiopsy for the Support of the Diagnosis of Gynaecological Cancer (IPPOKRATIS), December 2002 - December 2004.

\section{REFERENCES}

[1] J.A. Fayez, M.F. Vogel, "Comparision of different treatment methods of endometriomas by laparoscopy," Obstet. Gynecol., Vol. 78, pp. 660-665, 1991.

[2] R. Wenzl, R. Lehner, U. Vry, N. Pateisky, P. Sevelda, P. Husslein, "Three-dimensional video-endoscopy: clinical use in gynaecological laparoscopy," Lancet, Vol. 344, pp. 1621-1622, 1994.

[3] M.S. Neophytou, C.S. Pattichis, M.S. Pattichis, V. Tanos, E. Kyriacou, D. Koutsouris, "Multiscale Texture Feature Variability Analysis in Endoscopy Imaging Under Different Viewing Positions," Proc. II EFOMP Med. Conf. on Med. Phys., Limassol, Cyprus, 2004.

[4] M.S. Neophytou, C.S. Pattichis, M.S. Pattichis, V. Tanos, E. Kyriacou, S. Pavlopoulos, D. Koutsouris, "Texture Analysis of the Endometrium During Hysteroscopy: Preliminary Results," Proc. IEEE EMBS 04 Conf., San Francisco, USA, Sep. 2004.

[5] Web link: http://www.acmicorp.com

[6] Web link: http://www.pinnaclesys.com

[7] Web link: http://www.edundoptics.com

[8] B.G. Haskell, A. Puri, and N. Netravali, Digital Video: An Introduction to MPEG-2, Int. Thomson Publishing, New York 1997.

[9] Web link: http://www.mathworks.com

[10] P.M. Tjoa, M.S. Krishnan, "Feature extraction for the analysis of colon status from the endoscopic images," BioMedical Engineering OnLine, Apr. 2003. Available: http://www.biomedical-engineeringonline.com/content/2/1/9.

[11] R.M. Haralick, K. Shanmugam, I. Dinstein, "Texture Features for Image Classification," IEEE Trans. on Systems, Man., and Cybernetics, Vol. SMC-3, pp. 610-621, Nov. 1973. 